\title{
Binary operation based hard exudate detection and fuzzy based classification in diabetic retinal fundus images for real time diagnosis applications
}

\author{
Arun Pradeep ${ }^{1}$, X. Felix Joseph ${ }^{2}$ \\ ${ }^{1}$ Department of Electrionics and Communication Engineering, Noorul Islam University, India \\ ${ }^{2}$ Department of Electrical and Electronics Engineering, NICHE and Bulehora University, Ethiopia
}

\begin{tabular}{l} 
Article Info \\
\hline Article history: \\
Received May 2, 2019 \\
Revised Nov 22, 2019 \\
Accepted Nov 30, 2019 \\
\hline
\end{tabular}

\section{Keywords:}

Binary operations

Diabetic retinal image

Fuzzy logic

Hard exudates

Image processing

\begin{abstract}
Diabetic retinopathy (DR) is one of the most considerable reasons for visual impairment. The main objective of this paper is to automatically detect and recognize DR lesions like hard exudates, as it helps in diagnosing and screening of the disease. Here, binary operation based image processing for detecting lesions and fuzzy logic based extraction of hard exudates on diabetic retinal images are discused. In the initial stage, the binary operations are used to identify the exudates. Similarly, the RGB channel space of the DR image is used to create fuzzy sets and membership functions for extracting the exudates. The membership directives obtained from the fuzzy rule set are used to detect the grade of exudates. In order to evaluate the proposed approach, experiment tests are carriedout on various set of images and the results are verified. From the experiment results, the sensitivity obtained is $98.10 \%$, specificity is $96.96 \%$ and accuracy is $98.2 \%$. These results suggest that the proposed method could be a diagnostic aid for ophthalmologists in the screening for DR.
\end{abstract}

Copyright $\odot 2020$ Institute of Advanced Engineering and Science. All rights reserved.

\section{Corresponding Author:}

Arun Pradeep,

Department of Electronics and Communication Engineering,

Noorul Islam University,

Thuckalay, Kanyakumari District, Tamil Nadu, India.

Email: arunpradeep@msn.com

\section{INTRODUCTION}

Diabetic Retinopathy (DR), which is the real reason for visual deficiency, could be backed off by early recognition. Programmed screening frameworks help in recognizing the essential manifestations like exudates, which are because of the spillage of lipids from blood vessels. These lipids look like yellow shade on the retinal images. A skillful pre-processing of the images is essential for managing, magnificence and separation of assortments. A set of optimally adjusted morphological operators for exudate recognition on diabetic retinopathy patients' was proposed by Sopharak et.al. [1]. Ophthalmologists' hand-drawn groundtruths are used for comparison and validation of detected exudates. Sanchez et.al. [2, 3] presented an automatic image processing algorithm for the detection of hard exudates. In this work, the authors used a database containing 58 retinal images with different combinations of colour, brightness, and quality. The computational intelligence techniques are also used for the automated identification of exudate pathologies in retinopathy images [4]. The technique employs a dataset with 300 retinal images and the technique achieved $96 \%$ sensitivity. A morphology mean shift algorithm based approach for the detection of exudate lesions in color retinal images was introduced by Wisaeng et.al. [5]. The Mathematical Morphology Algorithm (MMA) is the key element in this method. Multiscale Amplitude-ModulationFrequency-Modulation (AM-FM) methods are useful for discriminating between the normal and pathological retinal images [6]. An automated diabetic retinopathy diagnosis system useful for the detection of various 
lesions of the retina including exudates, microaneurysms and hemorrhages get wide acceptance [7]. Many authors tried to develop more efficient DR detecting schemes [8-14].

To further enhance the detection accuracy, a combination of 3 methods, viz. Image binarization, Region of Interest (ROI) based segmentation and Morphological Reconstruction (MR) are proposed. [15]. Nidhal and Enas [16] developed a method for localizing, isolating the optic disk and then detecting the exudates. For localizing the optic disk, and treating the similarity between exudates and optic disk, they developed a new algorithm.

Inorder to improve the detection, gradient controlled fuzzy c means alsorithm are proposed [17]. For reducing the noise content in the original image, use of smoothening filters is also proposed in this work. Carla et.al. [18] presented an automated system for detection of exudates in the macula. This approach uses optimal thresholding of Instantaneous Amplitude (IA) components. Here, the IA components are extracted from multiple frequency scales for generating candidate exudate regions. [18]. New pre-processing methods which perform normalization, denoising, detect reflections and artifacts in the image were developed by Zhang et.al. [19]. In this work, authors developed a candidate's segmentation method which is based on mathematical morphology. Harangi and Hajdua [20] presented a way for automatic segmentation of exudates consisting of a candidate extraction step. Here, the extraction of possible exudate candidates is done by the use of grayscale morphology and their proper shape is determined by a Markovian segmentation model. A non-linear background elimination strategy for improved automatic exudates detection in retinal images was introduced by Akter et.al. [21]. Similar studies can be extensively found in literature [22-24]. For the computer assisted recognition, a hybrid algorithm using Migrating Bird Optimisation and Support Vector Machine (MB-SVM) classifiers has been proposed [25]. A four steps strategy for the detection was devoleped by Elbalaoui and Fakir [26]. It includes shifting the colour space, Optic Disc (OD) elimination, exudates segmentation and finally separation of exudates from the background.

\section{RESEARCH METHOD}

This paper proposes an algorithm which combines two different principles for detecting and extracting hard exudates from the given retinal images; they are binary operation and fuzzy logic. At first, mathematical binary operations are used to identify the exudates after pre-processing and optic disc elimination. The detail is shown in Figure 1.

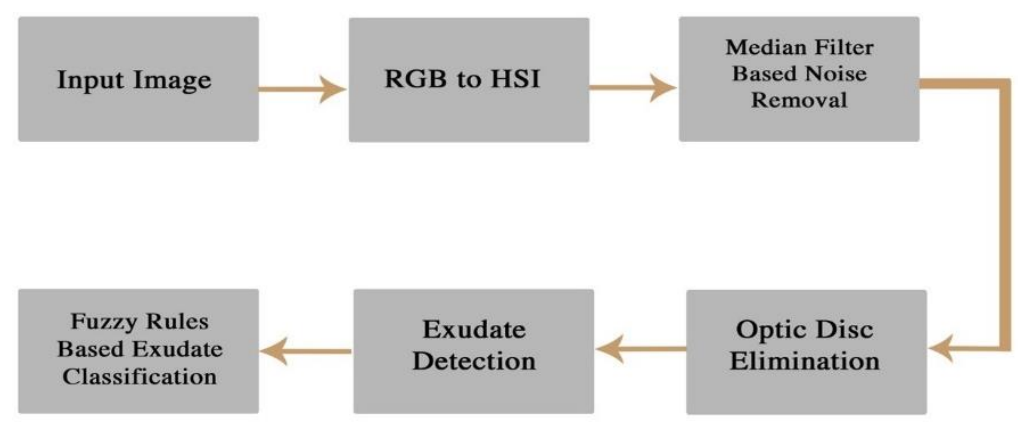

Figure 1. Proposed method

During pre-processing, the RGB color image is converted into HSI color space. Then the noise occurred in the image is eliminated by applying median filter over the intensity band. Following this noise removal, Contrast Limited Adaptive Histogram Equalization (CLAHE) method is applied to enhance the image based on the contrast value. This will prevent the saturation of the similar areas in the image. Some of the earlier research works used Gaussian function for noise removal. Figure 2 shows the pre-processing steps. The color conversion from RGB to HSI and I-band of the images are used for next level processing. If the intensity of exudates and optic disc are high, then the RGB color image is converted into HSI and proceded for further processing. The conversion RGB to HSI is a direct work and it doesn't need a conversion from RGB to grayscale. In order to eliminate the optic disc, it is considered that the optic disc is a component with largest circular shape that exists in the fundus images. In the second process the exudates are classified as soft and hard using fuzzy logic rules. The fuzzy rules are created using fuzzy sets obtained from the RGB fundus images. The fuzzy rules determine the availability of the hard exudates in the diabetic retinal images. 
There are some imperfections in binary images. Binary regions of the images can be produced by certain level of threshold values and are biased by texture and noise. Binary operations follow the objectives of eliminating the imperfections by considering them to form a structure of the image. It can be obtained from HSI image.

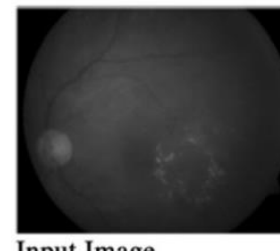
Input Image

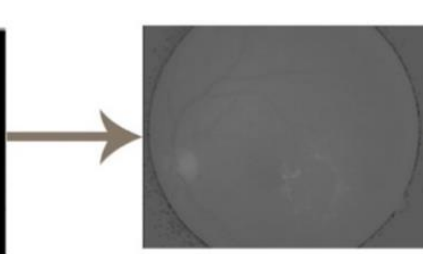

HSI image
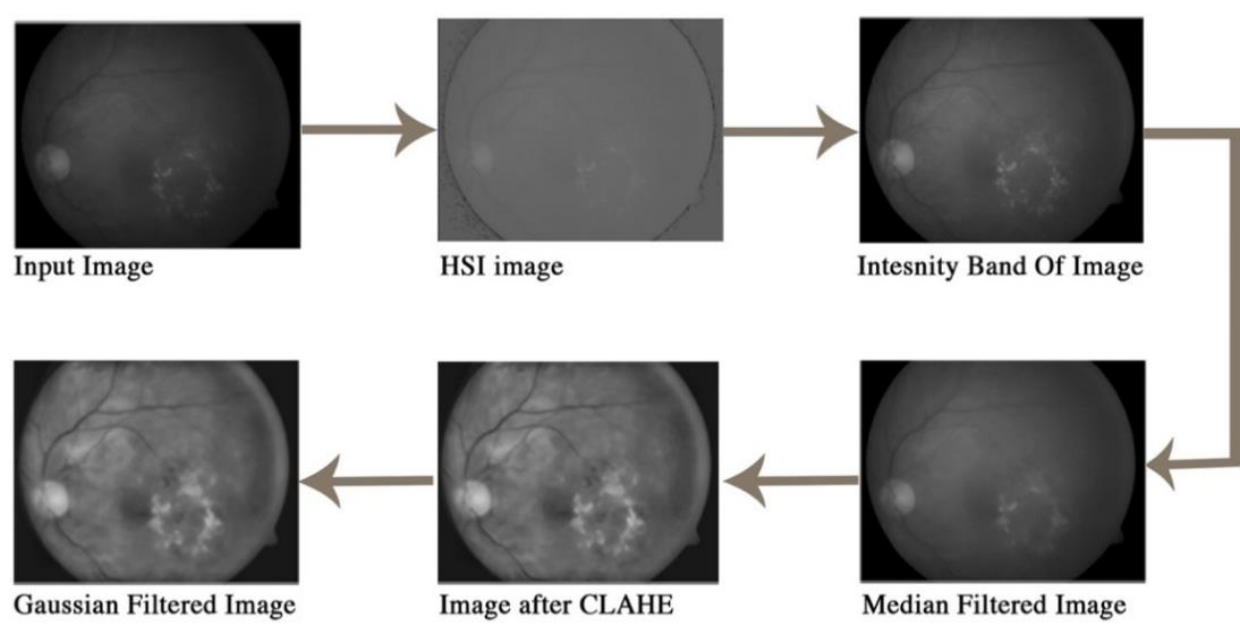

Image after CLAHE

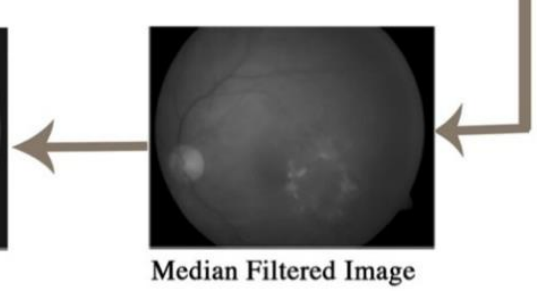

Figure 2. Result obtained from preprocessing stage

\subsection{Optic disc elimination}

Opening, closing, and erosion are some of the operations involved under binary and morphological operations. In this paper the closing operation is applied to get the structure of the disc on the input image $\mathbf{I}$. After closing operation, a simple threshold method is applied to get a binary image. The output image $\boldsymbol{\Omega}$ comprises of various connected regions such as $\boldsymbol{C}_{\boldsymbol{i}}$ and it can be represented as:

$$
\Omega=\bigcup_{k \in m} C_{k}, C_{i} \cap C_{j}=0, \forall i, j \in m, i \neq j
$$

Where, $\boldsymbol{m}$ changed from $\mathbf{1}$ to $\boldsymbol{k}, \boldsymbol{k}$ is the total number of components connected in the image. Comparing with the background, all the components are having more number of pixels and forms a circular shape that belongs to $\boldsymbol{C}_{\boldsymbol{i}}$. Optic disc is also included in $\boldsymbol{C}_{\boldsymbol{i}}$. Hence separating the component (optic disc) from $\boldsymbol{C}_{\boldsymbol{i}}$ is very easy and can be extracted effectively from all the other structures in the retinal image. The biggest region based component is called as $\boldsymbol{R}_{\boldsymbol{i}}$ available in $\boldsymbol{C}_{\boldsymbol{i}}$, where the compactness of $\boldsymbol{R}_{\boldsymbol{i}}$ can be measured using the following formula as:

$$
C\left(R_{i}\right)=4 \pi \frac{A\left(R_{i}\right)}{P^{2}\left(R_{i}\right)}
$$

Where, the number of pixels in the $\boldsymbol{i}^{\boldsymbol{t h}}$ region is denoted as $\boldsymbol{A}\left(\boldsymbol{R}_{\boldsymbol{i}}\right)$ and the pixels out of the region $\left(\boldsymbol{R}_{\boldsymbol{i}}\right)$ is $\boldsymbol{P}\left(\boldsymbol{R}_{\boldsymbol{i}}\right)$. In this paper in order to increase the efficiency, already proven methods such as P-tile method [16] and Nilblack's method [17, 18] are taken as the thresholding methods. The optic disc is considered as the largest connected component where it provides the highest values of compactness among these two methods. Since exudates and optic disc are having similar color and intensity, it is necessary to eliminate the optic disc to separate the exudates. Figure 3 shows the output of the image after applying the binary operation (closing) as OD eliminated including blood vessels.

OD is identified by the largest circular shape available on the connected components. In the Nilblack's method, 1.3 is considered as the weight factor. The region containing the optic disc is brighter than the other regions of the retinal image. From the entire image, the OD comprises of $2 \%$ of brightness and it is used to obtain the binary images which are shown in the output. The output shown in Figure 3 illustrates the binary operation based results. In order to isolate the optic disc, the Circular Hough Transformation (CHT) method is used. 

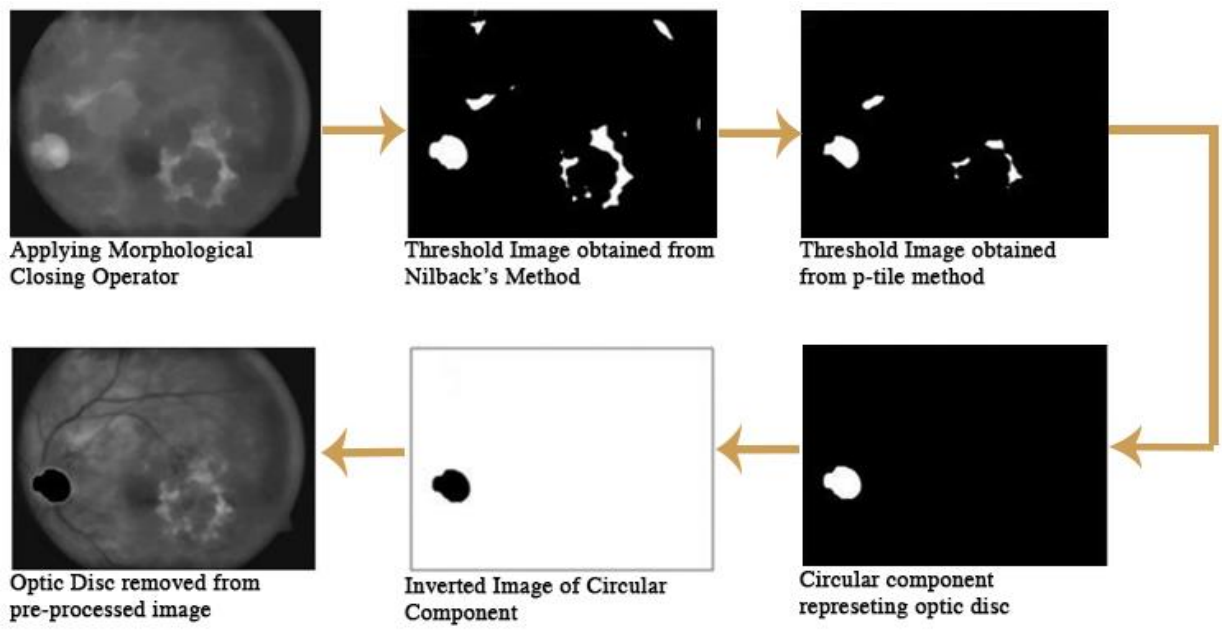

Figure 3. Optic disc elimination

\subsection{Detection of exudates}

After OD detection and elimination, further process of the proposed system is to identify and extract the hard exudates. For the direct detection of the hard exudates, binary closing operation with flat disc shaped structuring element using a 16-pixels radius is employed. This closing operation is applied after thresholding method. This operation includes the same level of contrast component blood vessels also. So, the standard deviation of the image is computed. In order to differentiate the components a triangle thresholding method is applied to detect the very brighter region in the image. From that, unwanted regions are removed by dilation operation from the thresholding image. After that, flood filling operation is applied on the holes to make the image ready for reconstruction from the binary operation. Finally, the difference image is taken between the output image and the intensity based original image (which is obtained from thresholding). The result of this threshold image is super-imposed on the real RGB image for extracting the exudates.

$$
\mathrm{I}_{3}(\mathrm{x})=\frac{1}{\mathrm{~N}-1} \sum_{\mathrm{i} \in \mathrm{W}(\mathrm{x})}\left(\mathrm{I}_{2}(\mathrm{i})-\overline{\mathrm{I}_{3}(\mathrm{x})}\right)^{2}
$$

Where, $\mathbf{W}(\mathbf{x})$ represents the pixels available in the sub-window, $\boldsymbol{N}$ represents the total number of pixels in $\mathbf{W}(\mathbf{x})$ and $\overline{\mathbf{I}_{\mathbf{3}}(\mathbf{x})}$ represents the mean value of the image $\mathbf{I}_{\mathbf{3}}(\mathbf{x})$ and $\mathbf{I}_{\mathbf{3}}$ represents the local contrast image.

\subsection{Fuzzy logic based hard exudate classification- creating fuzzy rules and membership functions}

Considering a collection of objects represented as $\boldsymbol{X}$, then a fuzzy set $\boldsymbol{A}$ is over $\boldsymbol{X}$ which is defined as the set of all ordered pairs $\boldsymbol{A}=\left\{\left(\boldsymbol{x}, \boldsymbol{\mu}_{\boldsymbol{A}}(\boldsymbol{x})\right) \mid \boldsymbol{x} \in \boldsymbol{X}\right\}$, where $\boldsymbol{\mu}_{\boldsymbol{A}}(\boldsymbol{x})$ is called as the set of membership functions related to the fuzzy set $\boldsymbol{A}$. The membership function automatically maps all the elements in the $\mathrm{X}$ to a membership degree among 0 to 1 such that $\boldsymbol{\mu}_{A}: \mathbf{X} \rightarrow[\mathbf{0}, \mathbf{1}]$.

Fuzzy Set: The set of all support of a fuzzy set $\boldsymbol{A}$ represents all the points including non-zero membership degree in $\boldsymbol{A}$ such that support $(\boldsymbol{A})=\left\{\boldsymbol{x} \in \boldsymbol{X} \mid \boldsymbol{\mu}_{\boldsymbol{A}}(\boldsymbol{x})>0\right\}$.

Defuzzification: The de-fuzzified values $\boldsymbol{x}^{*}$ is also called as crisp values and it can be represented by,

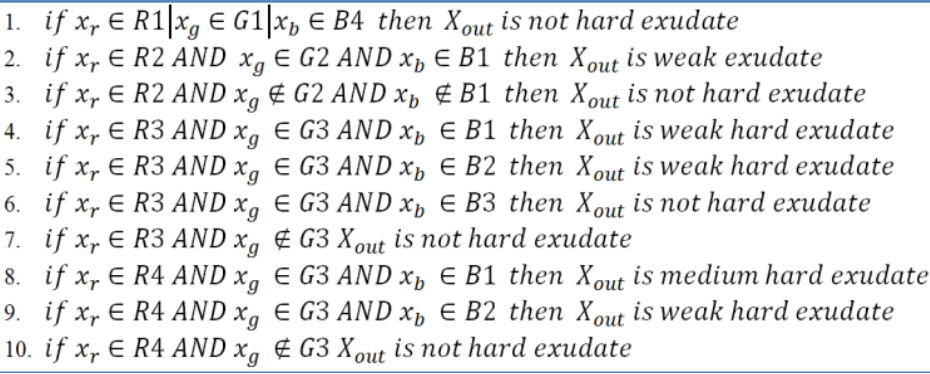

$$
x^{*}=\frac{\int_{s} x \mu_{i}(x) d x}{\int_{s} \mu_{i}(x) d x}
$$


Where $\boldsymbol{s}$ is represented as the support of the aggregated membership function $\boldsymbol{\mu}_{\boldsymbol{i}}(\boldsymbol{x})$.

The final and main process of the proposed approach is identifying the hard exudates using fuzzy logic. In order to create fuzzy set, the fuzzy membership function is obtained by RGB color channel of the retinal image. The three values representing the three different color channels which are represented as $x_{r}, x_{g}$ and $x_{b}$ are fed as input values into Fuzzy Interference System (FIS). In order to get the output for $x_{r}, x_{g}$ and $x_{b}$ with respect to the fuzzy rules, the fuzzy interference system gives a degree of membership to the output variable as $X_{\text {out }}$. The above described crisp values are obtained according to a centroid method used to compute the output. All the variables $x_{r}, x_{g}, x_{b}$ and $X_{\text {out }}$ are represented using the Gaussian member function as:

$$
\mu(x)=e^{-\left(\left(C_{i}-x\right)^{2}\left(2 \sigma i^{2}\right)\right)}
$$

There are five fuzzy sets which are derived for representing the output variables. The fuzzy result is counted on all the image elements in each exudate in the retinal images. The area of the hard exudates is determined by an average fuzzy value as $>0.25$. The output is also calculated in accordance to the percentage of the hard exudate's area. The fuzzy rules are mainly used for classifying the hard exudates. The crisp values of each hard exudate are calculated for classifying it. If the crisp value should be $>0.25$, then it is used to determine the hard exudates. Fuzzy output lesser than 0.25 is considered as soft or tiny exudates.

\section{RESULTS AND ANALYSIS}

The fuzzy rules and all the discussed procedure were implemented in MATLAB and results are verified. In this proposed method, dataset named DIARETDB0 and DIARETDB1 are used for implementing and testing. All the exudates are classified using crips value. For getting the accurate classification, hard exudates are used. Figure 4 represents the tiny sized hard exudates extracted. The entire data set is divided into two sub sets as training set and testing set. The features of extracted image are used for cross validation and to find out the misclassification.

In this paper 75 images are taken from the dataset DIARETDB0 for experiment and the total numbers of correctly classified images are counted. The soft, hard exudates are classified by more experiments and comparing the results of the proposed approach delivers a result given below in Table 1 . The performance can be evaluated by calculating the metrics True Positive Rate (TPR) and False Positive Rate (FPR). The TPR is the ratio of number of normal images identified to the total number of normal image and FPR is the ratio of number of wrongly obtained classifications to the total number of image to be classified. The accuracy, sensitivity and specificity are found out based on the formulas as given below:

$$
\begin{aligned}
& \text { Accuracy }=\frac{\text { True Negatives(TN)+True Positives }(\mathrm{TP})}{\text { True positives(TP)+False Positives(FP)+True Negatives(TN)+False Negatives(FN) }} \\
& \text { Sensitivity }=\frac{\mathrm{TP}}{\mathrm{TP}+\mathrm{FN}} \\
& \text { Specificity }=\frac{\mathrm{TN}}{\mathrm{TN}+\mathrm{FP}}
\end{aligned}
$$

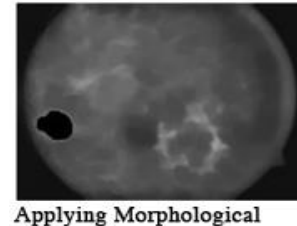
Applying Morphological Closing Operato

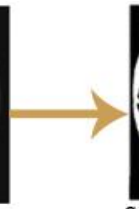

Standard Deviation and Thresholding using Triangle Method
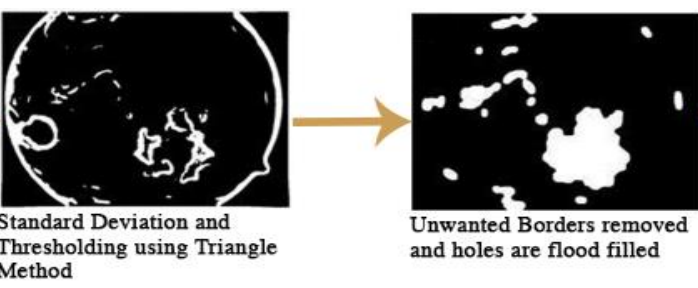
and holes are flood filled

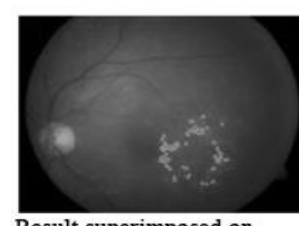

Result superimposed on Original Image
Thresholded Image

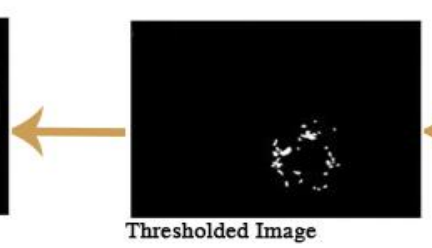

Figure 4. Exudates detection

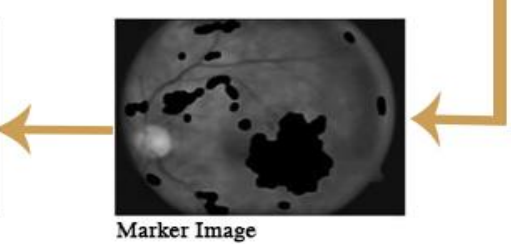


The total number of input images experimented and number of images classified by the proposed approach is given in the following Table 1 . The total number of images is 75 , in that, 50 images are having soft exudates and 25 images are having hard exudates. In order to obtain better results four-fold cross validation is applied and the obtained result is shown in Table 2. Detecting and differentiating exudates as soft, hard exudates are a hard task, and the obtained TPR, FPR values are comparatively better than the existing system. Also the efficacy of the proposed method can be analyzed using sensitivity and specificity metrics. Since the sensitivity and the specificity are comparatively better than the existing approaches, it is decided that the proposed approach is efficient. Also the performance of the proposed approach is evaluated by comparing it with the existing systems.

Table 1. Exudate Versus Non-Exudate Comparison Output

\begin{tabular}{lllll}
\hline Disease & Fold-1 & Fold-2 & Fold-3 & Fold-4 \\
\hline Exudates & 85.7 & 100.0 & 88.5 & 92.6 \\
Non-Exudates & 12.9 & 10.0 & 6.1 & 9.4 \\
\hline
\end{tabular}

Table 2. Performance Analysis in Terms of Sensitivity, Specificity and Accuracy

\begin{tabular}{lllll}
\hline Measures & Fold-1 & Fold-2 & Fold-3 & Fold-4 \\
\hline Sensitivity & 86.21 & 95.12 & 98.10 & 98.10 \\
Specificity & 87.0 & 98.10 & 96.96 & 96.96 \\
Accuracy & 86.66 & 96.66 & 98.33 & 98.33 \\
\hline
\end{tabular}

A large value of specificity means, the proposed algorithm does not extract the wrong portion as exudates and a low value represents the proposed algorithm is not efficient for extracting the exudates accurately. To determine the accurate exudates, the average crisp values are utilized by the fuzzy logic. The proposed approach extracts the hard exudate when the crisp value is $>0.09$. It promised that the proposed approach doesn't classify the soft exudates. In order to compute the performance of the proposed approach, Receiver Operator Characteristics (ROC) is generated. Here, an intermediate interval, [0, 1] is taken for locating the pixels which are potential true-negative regions. Figure 5 shows the plot of ROC analysis for the results obtained from real time experiment.

Only few images are taken from the real time image dataset since processing the entire dataset takes long execution time. Therefore, out of 200 images, 30 images are used in training process and the remaining 170 images are used for testing process. To evaluate the performance in real time clinical images, the testing images are collected from Dr.Bhejan Singh Eye Hospital, Nagarcoil, Tamilnadu. The best operating point on this curve is at a sensitivity of $98.10 \%$ at a specificity of $96.96 \%$.

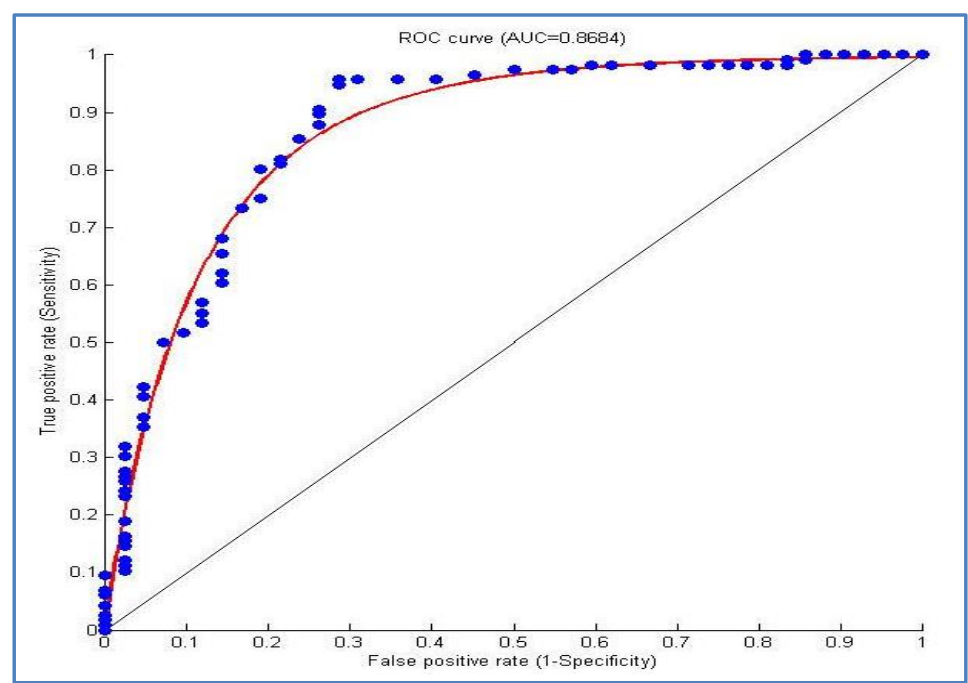

Figure 5. ROC curve for testing set of exudates at image level 


\section{CONCLUSION}

Identification, detection, extraction and classification of hard exudates from diabetic retinal image are done. For this purpose, the image is preprocessed, normalized, detected, extracted and classified. It can be obtained through color conversion, median filtering, binary operations and fuzzy logic. Comparing with other methods the accurate determination of hard exudates is done by fuzzy rules on the retinal image data. In this paper a sequence of image processing steps with fuzzy rules is applied to determine the size and intensity of the exudates. The output is automatically mapped by the Fuzzy Interference System. The image intensity and size (area) are the parameters used to compute the exudates and a threshold based adjustment is done by a ROC analysis. The obtained results and the ROC analysis depicts that the proposed approach is more efficient. The obtained sensitivity is $98.10 \%$, Specificity is $96.96 \%$ and Accuracy is $98.2 \%$. These results suggest that the proposed method could be a diagnostic aid for ophthalmologists in the screening for DR. In future the proposed work is extended to identifying the other pathological information such as occlusion, hemorrhages, macula edema and red spots.

\section{REFERENCES}

[1] A. Sopharak et.al., "Automatic Detection of Diabetic Retinopathy Exudates from Non-Dilated Retinal Images using Mathematical Morphology Methods," Comput Med Imaging Graph, vol. 32, pp. 720-727, 2008.

[2] C. I. Sanchez et.al., "A Novel Automatic Image Processing Algorithm for Detection of Hard Exudates Based on Retinal Image Analysis," Med EngPhys, vol. 30, pp. 350-357, 2008.

[3] C. I. Sanchez et.al., "Retinal Image Analysis Based on Mixture Models to Detect Hard Exudates," Med Imag Anal, vol. 13, pp.650-668, 2009.

[4] A. Sareh et.al., "Computational Intelligent Based Approach for Detection Ofexudates in Diabetic Retinopathy Images,” IEEE Trans InfTechnol Biomed, vol. 13, pp. 535-545, 2009.

[5] K. Wisaeng and W. Sa-Ngiamvibool, "Exudates Detection using Morphology Mean Shift Algorithm in Retinal Images," in IEEE Access, vol. 7, pp. 11946-11958, 2019.

[6] C. Agurto et.al., "A Multi-scale AM-FM Methods for Diabetic retinopathy Lesion Detection," IEEE Trans Med Imaging, vol. 29, pp. 502-512, Feb 2010.

[7] N. Singh and R. C. Tripathi, "Automated Early Detection of Diabetic Retinopathy using Image Analysis Techniques,” Int. J. Comput.Appl, vol. 8, pp. 18-23, 2010.

[8] M. Esmaeili et.al., "Automatic Detection of Exudates and Optic Disk in Retinalimages using Curvelet Transform," IET Image Process, vol.6, pp. 1005-1013, 2012.

[9] N. G. Ranamuka and R. G. N. Meegama, "Detection of Hard Exudates from Diabetic Retinopathy Images using Fuzzy Logic,” in IET Image Processing, vol. 7, no. 2, pp. 121-130, March 2013.

[10] M. G. F. Eadgahi and H. Pourreza, "Localization of Hard Exudates in Retinal Fundus Image by Mathematical Morphology Operations," 2012 2nd International eConference on Computer and Knowledge Engineering (ICCKE), Mashhad, pp. 185-189, 2012.

[11] L. Giancardo et.al., "Exudate Based Diabetic Macularedema Detection in Fundus Images using Publicly Availabledata Sets," Med ImageAnal, vol. 16, pp. 216-226, 2012.

[12] F. Amel et.al., "Improvement of Thehard Exudates Detection Method used for Computer-Aideddiagnosis of Diabetic Retinopathy," Int J of Imag, Graph SignProcess, vol. 4, pp.19-27, 2012.

[13] H. B. Kekre et.al., "Hybrid Approach Fordetection of Hard Exudates," Int J of Advanc Comp SciAppl, vol. 4, pp. 250-255, 2013.

[14] Bu Wei et.al., "Hierarchicaldetection of Hard Exudates in Color Retinal Images," J Soft, vol. 8, pp. 2723-2732, 2013.

[15] S. Srenget et. al., "Automatic Exudate Extraction for Early Detection of Diabetic Retinopathy," 2013 International Conference on Information Technology and Electrical Engineering (ICITEE), Yogyakarta, pp. 31-35, 2013.

[16] K. Nidhal and E. H. Al-Saadi, "Automaticdetection of Exudates in Retinal Images," Int J Comp Sci, vol. 10, pp. 237242, 2013.

[17] R. Ravindraiah and J. L. MazharIqbal, "Hard Exudates Detection Inproliferative Diabetic Retinopathy using Gradient Controlledfuzzy C Means Clustering Algorithm," GESJ Comp. Sci. and Telecomm., vol. 44, pp. 27-31, 2014.

[18] C. Agurto et al., "A Multiscale Optimization Approach to Detect Exudates in the Macula," in IEEE Journal of Biomedical and Health Informatics, vol. 18, no. 4, pp. 1328-1336, July 2014.

[19] Zhang X., Thibault G., Deceniere E., Marcotegui B., Danno R., Cazuguel G., Quellec G., Lamard M., Massin P., Chabouis A., Victor Z., "Erginary A. Exudate Detection in Color Retinalimages for Mass Screening of Diabetic Retinopathy," Med ImaAnal, vol. 18, pp. 1026-1043, 2014.

[20] B. Harangi and A. Hajdu, "Detection of Exudates in Fundus Images using a Markovian Segmentation Model," 2014 36th Annual International Conference of the IEEE Engineering in Medicine and Biology Society, Chicago, IL, pp. 130-133, 2014.

[21] M. Akter et.al., "An Improvedmethod of Automatic Exudates Detection in Retinal Images," Int J Innov Res Elect, Electron, Instrument ContEngin, vol. 2, pp. 1514- 1516, 2014. 
[22] S. Kalashree and K.S. Sowmya, "A Novel Algorithm for Exudates Detection using MATLAB," Intern J. Advan. Res. Comp. Sci. Soft. Eng., vol. 5, pp. 276-280, 2015.

[23] R. Bhargavi and V. Rajesh,"Exudate Detection and Featureextraction using Active Contour Model and Sift in Colorfundus Images," ARPN J. Eng. Appl. Sci., vol. 10, pp. 2362-2365, 2015.

[24] P. M. Rokade, "Automatic Hard Exudates Detection Frommulti-Resolution Retinal Images using Haar Duel Tree Wavelettransform," Int. J. Comp. Sci. Tren. Technol., vol. 3, pp. 238-245, 2015.

[25] R. Badgujar and P. Deore, "MBO-SVM-Based Exudate Classification in Fundus Retinal Images of Diabetic Patients," Comp Methods in Biomecs and Biomed Eng: Img\&Visualiz, vol. 7, pp. 195-206, 2019.

[26] A. Elbalaoui and M. Fakir, "Exudates Detection in Fundus Images using Mean-Shift Segmentation and Adaptive Thresholding," Comp. Meth. in Biomechand Biomed. Eng. Imag. \& Visualiz., vol. 7, pp. 145-153, 2019.

\section{BIOGRAPHIES OF AUTHORS}
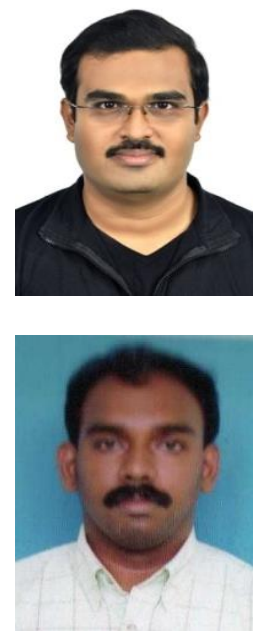

Arun Pradeep is a Research Scholar at Noorul Islam University, Tamil Nadu. He received his B.Tech in Electronics and Communication Engg. in 2009 and M.tech in Embedded Systems in 2012. He was an Assistant professor in Electronics and Communication Engineering. at Axis College of Engineering, Thrissur, Kerala during the period 2012 to 2018. His research interest field is Medical Image processing.

X. Felix Joseph is an Assistant Professor at Bule hora University, Ethiopia. Dr. Felix Joseph has more than 14 years of experience in the teaching field in the area of Electrical and Electronics, $\mathrm{He}$ has attended more than 60 short term training programs including two weeks STTP, one week seminar,one day workshops, and two weeks training programs. Currently he is guiding seven research scholars and published more than 10 papers in international journals and conferences. His areas of interests are soft computing applications in digital signal processing, digital image processing and power electronics. His favorite subject is neural network, which is one of the tool used in soft computing techniques. 